\title{
Linear Discrete Pursuit Game Problem with Total Constraints
}

\author{
Atamurat Kuchkarov, ${ }^{1}$ Gafurjan Ibragimov, ${ }^{2}$ and Akmal Sotvoldiev ${ }^{1}$ \\ ${ }^{1}$ Institute of Mathematics of Uzbekistan, 29 Dorman yuli Street, 100125 Tashkent, Uzbekistan \\ ${ }^{2}$ Institute for Mathematical Research and Department of Mathematics, Faculty of Science (FS), Universiti Putra Malaysia, \\ 43400 Serdang, Selangor, Malaysia \\ Correspondence should be addressed to Gafurjan Ibragimov; gafur@science.upm.edu.my
}

Received 8 March 2013; Revised 28 May 2013; Accepted 28 May 2013

Academic Editor: Valery Y. Glizer

Copyright (C) 2013 Atamurat Kuchkarov et al. This is an open access article distributed under the Creative Commons Attribution License, which permits unrestricted use, distribution, and reproduction in any medium, provided the original work is properly cited.

We study a linear discrete pursuit game problem of one pursuer and one evader. Control vectors of the players are subjected to total constraints which are discrete analogs of the integral constraints. By definition pursuit can be completed in the game if there exists a strategy of the pursuer such that for any control of the evader the state of system $z(t)$ reaches the origin at some time. We obtain sufficient conditions of completion of the game from any initial position of the state space. Strategy of the pursuer is defined as a function of the current state of system and value of control parameter of the evader.

\section{Introduction}

Pursuit and evasion differential game problems with integral constraints for continuous time dynamical systems were investigated in many works, (see, for example, [1-14]). Such constraints represent the constraints on resource, energy, fuel, and so forth. Discrete analog of integral constraint is called total constraint.

There are many works on discrete controlled systems where controls are subjected to total or geometric constraints. Sirotin [15] studied some conditions of 0-controllability and asymptotical controllability of discrete time linear systems. In this work, the control is bounded in Holder norm. In particular, he obtained necessary and sufficient conditions of 0 -controllability and asymptotical controllability of the system.

Sazanova [16] studied the problem of steering the state of a linear discrete system from the given point at a given time to another given point at a given time by a control which minimizes a given functional. She constructed an optimal program control.

However, discrete linear pursuit games with total constraints on control vectors of players were studied in a few works, for example, [17-20]. Satimov et al. [19] examined linear discrete pursuit games of many objects under total constraints on controls of players. Game is described by the following systems:

$$
\begin{array}{r}
z_{i}(n+1)=C_{i} z_{i}(n)+u_{i}(n)-v(n), \\
z_{i}(0)=z_{i 0}, \quad i=1, \ldots, m .
\end{array}
$$

It is assumed that the eigenvalues of matrices $C_{i}$ are real. Sufficient conditions of completion of pursuit were obtained.

The paper of Satimov and Ibragimov [20] was also devoted to discrete time pursuit games of several players described by the same linear systems. They obtained sufficient conditions of completion of the game for any matrices $C_{i}$. It should be noted that in case of one pursuer these conditions are also necessary. In particular, they obtained necessary and sufficient conditions of 0 -controllability when the coefficient matrix of control is an identity matrix.

Azamov and Kuchkarov [17] obtained necessary and sufficient conditions of solvability of both the 0 -controllability problem and pursuit problem for a linear discrete system when the control parameter of pursuer is subjected to geometric constraint and that of evader is subjected to total constraint.

In the monograph of Azamov [21], the fundamentals of theory of discrete time pursuit and evasion dynamic games 
are given systematically. The main notions of the theory of discrete games were explained by interesting examples.

In the present paper, we study a linear discrete pursuit game problem described by the linear equation with constant coefficients of general form. Controls of both pursuer and evader are subjected to total constraints. We give sufficient conditions of completion of pursuit from any initial position.

\section{Statement of the Problem}

We consider a discrete pursuit game described by the following equation:

$$
z(t+1)=A z(t)+B u(t)+C v(t)
$$

where $t$ is a step number which belongs to the set of nonnegative integers, $z(t) \in \mathbb{R}^{n}, A, B$, and $C$ are $n \times n, n \times m$, and $n \times l$ constant matrices, respectively, $u \in \mathbb{R}^{m}$ is the control parameter of pursuer, and $v \in \mathbb{R}^{l}$ is that of evader.

Definition 1. Sequences $u(\cdot): N \rightarrow \mathbb{R}^{m}$ and $v(\cdot): N \rightarrow \mathbb{R}^{l}$, subjected to the following total constraints:

$$
\begin{aligned}
& \|u(\cdot)\|_{l_{p}}=\left(\sum_{t=0}^{\infty}|u(t)|^{p}\right)^{1 / p} \leq \rho, \\
& \|v(\cdot)\|_{l_{p}}=\left(\sum_{t=0}^{\infty}|v(t)|^{p}\right)^{1 / p} \leq \sigma,
\end{aligned}
$$

are referred to as the controls of the pursuer and evader, respectively, where $N$ is the set of nonnegative integers, $\rho>0$ and $\sigma \geq 0$ are given numbers, and $p>1$.

The conditions (3) and (4) are the discrete analogs of integral constraints, and they are called total constraints.

Definition 2. A function $U(z, v), U: \mathbb{R}^{n} \times \mathbb{R}^{l} \rightarrow \mathbb{R}^{m}$, is called strategy of the pursuer. The triple $\left(z_{0}, U, v(\cdot)\right)$ consisting of a given initial point $z_{0}$, the strategy $U$, and a control of the evader $v(\cdot)$ generates the unique trajectory $z(t)$ defined by the following formula:

$$
\begin{array}{r}
z(t+1)=A z(t)+B U(z(t), v(t))+C v(t), \\
z(0)=z_{0} .
\end{array}
$$

The sequence $u(t)=U(z(t), v(t)), t \in N$, is referred to as the realization of the strategy $U$. The strategy of the pursuer $U$ is called admissible if all its realizations with fixed $z_{0}$ satisfy the condition (3).

Definition 3. Pursuit is said to be completed in the game (2)(4) from the initial position $z_{0}$ if there exists a strategy of the pursuer $U$ such that for any control of the evader $v(\cdot)$ the trajectory generated by $z_{0}, U$, and $v(\cdot)$ satisfies the condition $z(t)=0$ at some $t, t \in N$.

The aim of the pursuer is to realize the equality $z(t)=$ 0 as earlier as possible. Thus we deal with pursuit problem.
The pursuer uses a strategy, and the evader uses any control $v(\cdot)$. By Definition 2 at any time $t$ the value of the strategy is constructed based on the state $z(t)$ and value of the control parameter of the evader $v(t)$.

Problem 1. Find the conditions under which pursuit can be completed in the game (2)-(4) from any initial position.

\section{Main Result}

To prove the main result, we first prove two lemmas. Let $\lambda_{1}, \lambda_{2}, \ldots, \lambda_{n}$ be the eigenvalues of the matrix $A$. Denote

$$
\begin{gathered}
\beta=\max _{1 \leq i \leq n}\left|\lambda_{i}\right|, \quad\|C\|=\max _{|z|=1}|C z|, \\
q=\frac{p}{p-1} .
\end{gathered}
$$

Let $V$ be the set of all sequences $v(\cdot)$ that satisfy the constraint (4).

Lemma 1. If $\beta<1$, then

(a) for all $t=1,2, \ldots$ the set

$$
X(t)=\left\{\sum_{i=0}^{t} A^{i} C v(t-i) \mid v(\cdot) \in V\right\}
$$

is contained in a ball $S_{r}^{n}=\left\{x \in \mathbb{R}^{n}|| x \mid \leq r\right\}$;

(b) for any triple consisting of $v(\cdot) \in V, \varepsilon>0$, and $t_{0} \epsilon$ $\{0,1,2, \ldots\}$ there exists a number $t^{*}, t^{*}>t_{0}$, such that

$$
\left|\sum_{i=0}^{t^{*}} A^{i} C v\left(t^{*}-i\right)\right| \leq \varepsilon
$$

Proof. Since $\beta<1$, then $\left\|A^{t}\right\| \rightarrow 0$ as $t \rightarrow \infty$; therefore there exists a positive integer $h$, such that $\mu \stackrel{\text { def }}{=}\left\|A^{h}\right\|<1$. Then according to the property of norm, we have

$$
\left\|A^{h k}\right\| \leq\left\|A^{h}\right\|^{k}<\mu^{k}, \quad k=1,2, \ldots
$$

Clearly,

$$
\begin{array}{r}
\left|\sum_{i=0}^{k h} A^{i} C v(k h-i)\right| \\
\quad=\left|\sum_{i=0}^{k} A^{h i} \sum_{j=0}^{h-1} A^{j} C v((k-i) h-j)\right| \\
\leq \sum_{i=0}^{k}\left\|A^{h i}\right\| \sum_{j=0}^{h-1}\left(\left\|A^{j}\right\| \cdot\|C\| \cdot|v((k-i) h-j)|\right), \\
k=1,2, \ldots
\end{array}
$$


Using the Holder inequality, we obtain for any $v(\cdot) \in V$ that

$$
\begin{aligned}
& \sum_{j=0}^{h-1}\left\|A^{j}\right\| \cdot|v((k-i) h-j)| \\
& \leq\left(\sum_{j=0}^{h-1}\left\|A^{j}\right\|^{q}\right)^{1 / q}\left(\sum_{j=0}^{h-1}|v((k-i) h-j)|^{p}\right)^{1 / p} \\
& \leq a \sigma, \quad k=1,2, \ldots ; a=\left(\sum_{j=0}^{h-1}\left\|A^{j}\right\|^{q}\right)^{1 / q} .
\end{aligned}
$$

It follows from (9)-(11) that

$$
\begin{aligned}
\left|\sum_{i=0}^{k h} A^{i} C v(k h-i)\right| & \leq a \sigma\|C\| \sum_{i=0}^{k} \mu^{i}=a \sigma\|C\| \frac{1-\mu^{k+1}}{1-\mu} \\
& \leq \frac{a \sigma\|C\|}{1-\mu}
\end{aligned}
$$

for all $k=1,2, \ldots$. Let $r=a \sigma\|C\|(1-\mu)^{-1}$. It is easy to check that if $0<t_{1}<t_{2}$, then $X\left(t_{1}\right) \subset X\left(t_{2}\right)$. Therefore, the inequality (12) implies that

$$
\left|\sum_{i=0}^{t} A^{i} C v(t-i)\right| \leq r, \quad t=1, \ldots, k h .
$$

Since (12) holds for any positive integer $k$, then the statement (a) of Lemma 1 follows.

We now turn to the statement (b) of the lemma. Assume the contrary: there exist a control $v(\cdot) \in V$ and positive numbers $\varepsilon, t_{0}$ such that

$$
\left|\sum_{i=0}^{t} A^{i} C v(t-i)\right|>\varepsilon, \quad \forall t \geq t_{0} .
$$

It follows from (9) that

$$
\begin{array}{r}
\max _{u \in S_{r}^{n}}\left|A^{h t} u\right| \leq \max _{u \in S_{r}^{n}}\left(\left\|A^{h t}\right\| \cdot|u|\right) \leq \mu^{t} r, \\
t=0,1,2, \ldots
\end{array}
$$

Since $0 \leq \mu<1$, then we can conclude that there exists a positive integer $T, T \geq t_{0}+1$, such that

$$
\max _{u \in S_{r}^{n}}\left|A^{t} u\right| \leq \frac{\varepsilon}{2} \quad \forall t \geq T .
$$

Clearly, (14) implies that

$$
\begin{gathered}
\varepsilon<\left|\sum_{i=0}^{(k+1) T-1} A^{i} C v((k+1) T-1-i)\right| \leq I_{1}+I_{2}, \\
I_{1}=\left|\sum_{i=0}^{T-1} A^{i} C v((k+1) T-1-i)\right|, \\
I_{2}=\left|A^{T} \sum_{i=T}^{(k+1) T-1} A^{i-T} C v((k+1) T-1-i)\right| .
\end{gathered}
$$

Setting $j=i-T$, we get

$$
\begin{gathered}
\sum_{i=T}^{(k+1) T-1} A^{i-T} C v((k+1) T-1-i) \\
=\sum_{i=0}^{k T-1} A^{j} C v(k T-1-j) .
\end{gathered}
$$

According to the part (a) of Lemma 1, this vector belongs to $S_{r}^{n}$. Then by (16), $I_{2} \leq \varepsilon / 2$. Hence, from (17), we obtain that $\varepsilon<I_{1}+(\varepsilon / 2)$. Therefore, using the Holder inequality yields

$$
\frac{\varepsilon}{2}<I_{1} \leq b\left(\sum_{i=0}^{T-1}|v((k+1) T-1-i)|^{p}\right)^{1 / p},
$$

where $b=\left(\sum_{i=0}^{T-1}\left\|A^{i} C\right\|^{q}\right)^{1 / q}$. This gives

$$
\sum_{i=0}^{T-1}|v((k+1) T-1-i)|^{p}>\left(\frac{\varepsilon}{2 b}\right)^{p} .
$$

Substituting $j=(k+1) T-1-i$ and then replacing $j$ by $i$ again, we obtain

$$
\sum_{i=k T}^{(k+1) T-1}|v(i)|^{p}>\left(\frac{\varepsilon}{2 b}\right)^{p}, \quad \forall k=1,2, \ldots
$$

We conclude from these inequalities that

$$
\sum_{i=T}^{(k+1) T-1}|v(i)|^{p}>k\left(\frac{\varepsilon}{2 b}\right)^{p}, \quad k=1,2, \ldots
$$

According to (4) for any $k$, the left hand side of this inequality is bounded by $\sigma^{p}$, while the right hand side $\rightarrow \infty$ as $k \rightarrow \infty$. Contradiction. The proof of Lemma 1 is complete.

Lemma 2. If $\beta<1$, then for any $v(\cdot) \in V$ the solution $z(\cdot)=$ $z\left(\cdot, v(\cdot), z_{0}\right)$ of the system

$$
z(t+1)=A z(t)+C v(t), \quad z(0)=z_{0},
$$

satisfies the equality

$$
\gamma\left(z\left(\cdot, v(\cdot), z_{0}\right)\right)=\inf _{t \geq 0}\left|z\left(t, v(\cdot), z_{0}\right)\right|=0 .
$$

Proof. Let $v(\cdot) \in V$ and $\varepsilon$ be an arbitrary positive number. Since $\beta<1$, then the system $z(t+1)=A z(t)$ is asymptotically stable. Therefore for any $z_{0}$ there exists a number $t_{1}$ such that

$$
\left|A^{t} z_{0}\right|<\frac{\varepsilon}{2}, \quad t \geq t_{1} .
$$

Next, by Lemma 1 there exists a number $t_{2}\left(t_{2} \geq t_{1}\right)$ such that

$$
\left|\sum_{i=0}^{t_{2}} A^{i} C v\left(t_{2}-i\right)\right| \leq \frac{\varepsilon}{2}
$$


Using (25) and (26), we have

$$
\begin{aligned}
\gamma(z( & \left.\left.t, v(\cdot), z_{0}\right)\right) \\
& \leq\left|z\left(t_{2}, v(\cdot), z_{0}\right)\right| \\
& =\left|A^{t_{2}} z_{0}+\sum_{i=0}^{t_{2}} A^{i} C v\left(t_{2}-i\right)\right| \\
& \leq\left|A^{t_{2}} z_{0}\right|+\left|\sum_{i=0}^{t_{2}} A^{i} C v\left(t_{2}-i\right)\right| \\
& <\frac{\varepsilon}{2}+\frac{\varepsilon}{2}=\varepsilon .
\end{aligned}
$$

As the number $\varepsilon$ is arbitrary, then $\gamma\left(z\left(t, v(\cdot), z_{0}\right)\right)=0$, and Lemma 2 follows.

Theorem 3. If $\beta<1$ and $\operatorname{dim} B S_{1}^{m}=n$, then pursuit can be completed in the game (2)-(4) from any initial position $z_{0} \in$ $\mathbb{R}^{n}$.

Proof. Define the strategy of the pursuer as follows:

$$
B U(z, v)= \begin{cases}0, & \text { if } A z+C v \notin B S_{\rho}^{m}, \\ -A z-C v, & \text { if } A z+C v \in B S_{\rho}^{m} .\end{cases}
$$

Since $\operatorname{dim} B S_{1}^{m}=n$, the set $B S_{\rho}^{m}$ contains a ball $S_{\delta}^{n}$ of radius $\delta>0$ of the space $\mathbb{R}^{n}$. It follows from Lemma 2 that for every control $v(\cdot) \in V$ there exists a step $t \in N$ such that $\mid A z(t)+$ $C v(t) \mid \leq \delta$. Consequently, for some $\tau$

$$
A z(\tau)+C v(\tau) \in B S_{\rho}^{m} .
$$

Then by (28) we have $B U(z(\tau), v(\tau))=-A z(\tau)-C v(\tau)$, and therefore

$$
z(\tau+1)=A z(\tau)+B U(z(\tau), v(\tau))+C v(\tau)=0 .
$$

This proves the theorem.

We now give an illustrative example.

Example 4. Consider the following discrete system in $\mathbb{R}^{2}$ :

$$
\begin{aligned}
z(t+1)= & {\left[\begin{array}{cc}
0.3 & -0.4 \\
0.4 & 0.3
\end{array}\right] z(t) } \\
& +\left[\begin{array}{ccc}
1 & 2 & 0 \\
-1 & 1 & 3
\end{array}\right] u(t)+\left[\begin{array}{cccc}
1 & 2 & -1 & 3 \\
3 & 1 & 4 & 5
\end{array}\right] v(t),
\end{aligned}
$$

where $z(t) \in \mathbb{R}^{2}, u(t)=\left(u_{1}(t), u_{2}(t), u_{3}(t)\right)$, and $v(t)=$ $\left(v_{1}(t), v_{2}(t), v_{3}(t), v_{4}(t)\right)$. Eigenvalues of the matrix

$$
A=\left[\begin{array}{cc}
0.3 & -0.4 \\
0.4 & 0.3
\end{array}\right]
$$

are $\lambda_{1,2}=0.3 \pm 0.4 i$, and $\left|\lambda_{1,2}\right|=0.5<1$. Next, it is not difficult to verify that $\operatorname{dim} B S_{1}^{3}=2$, where

$$
B S_{1}^{3}=\left\{B \xi \mid \xi=\left(\xi_{1}, \xi_{2}, \xi_{3}\right), \xi_{1}^{2}+\xi_{2}^{2}+\xi_{3}^{2} \leq 1\right\} .
$$

Thus, all hypotheses of Theorem 3 are satisfied, and therefore pursuit can be completed from any initial position $z_{0} \in \mathbb{R}^{2}$.

\section{Conclusion}

In the present paper, we have studied a linear discrete pursuit game problem under total constraints. We have obtained conditions under which pursuit starting from any initial position can be completed in a finite number of steps. We have described a strategy for the pursuer which is constructed based on the information about the position $z(t)$ and value of the control parameter of the evader $v(t)$ at each step $t$. It should be noted that the resource of the pursuer $\rho$ does not need to be greater than that of the evader $\sigma$.

\section{Acknowledgment}

The present research was partially supported by the National Fundamental Research Grant Scheme (FRGS) of Malaysia, 01-01-13-1228FR.

\section{References}

[1] A. Ya. Azimov, "Linear differential pursuit game with integral constraints on the control," Differential Equations, vol. 11, pp. 1283-1289, 1975.

[2] A. A. Chikrii and A. A. Belousov, "On linear differential games with integral constraints," Memoirs of Institute of Mathematics and Mechanics, Ural Division of RAS. Ekaterinburg, vol. 15, no. 4, pp. 290-301, 2009 (Russian).

[3] P. B. Gusiatnikov and E. Z. Mohon'ko, "On $l_{\infty}$-escape in a linear many-person differential game with integral constraints," Journal of Applied Mathematics and Mechanics, vol. 44, no. 4, pp. 436-440.

[4] G. I. Ibragimov, A. A. Azamov, and M. Khakestari, "Solution of a linear pursuit-evasion game with integral constraints," ANZIAM Journal. Electronic Supplement, vol. 52, pp. E59-E75, 2010.

[5] G. I. Ibragimov, M. Salimi, and M. Amini, "Evasion from many pursuers in simple motion differential game with integral constraints," European Journal of Operational Research, vol. 218, no. 2, pp. 505-511, 2012.

[6] G. I. Ibragimov and N. Yu. Satimov, "A multiplayer pursuit differential game on a closed convex set with integral constraints," Abstract and Applied Analysis, vol. 2012, Article ID 460171, 12 pages, 2012.

[7] R. Isaacs, Differential Games. A Mathematical Theory with Applications to Warfare and Pursuit, Control and Optimization, New York, NY, USA, 1967.

[8] N. N. Krasovskii, The Theory of Motion Control, Nauka, Moscow, Russia, 1968.

[9] A. V. Mesencev, "Sufficient conditions for evasion in linear games with integral constraints," Doklady Akademii Nauk SSSR, vol. 218, pp. 1021-1023, 1974.

[10] M. S. Nikolskii, "The direct method in linear differential games with integral constraints," Controlled Systems, IM, IK, SO AN SSSR, no. 2, pp. 49-59, 1969.

[11] L. S. Pontryagin, Selected Scientific Papers, vol. 2, Nauka, Moscow, Russia, 1988.

[12] B. N. Pshenichnii and V. V. Ostapenko, Differential Games, Naukova Dumka, Kiev, Russia, 1992.

[13] N. Yu. Satimov, Methods for Solving a Pursuit Problem in the Theory of Differential Games, NUUz Press, Tashkent, Uzbekistan, 2003, in Russian. 
[14] V. N. Ushakov, "Extremal strategies in differential games with integral constraints," Prikladna Matematika I. Mehanika, vol. 36, no. 1, pp. 15-23, 1972.

[15] A. N. Sirotin, "On null-controllable and asymptotically nullcontrollable finitedimensional linear systems with controls bounded in the Holder norms of control," Automation and Remote Control, vol. 60, no. 11, part 1, pp. 1729-1738, 1999.

[16] L. A. Sazanova, "Optimal control of linear discrete systems," Proceedings of the Steklov Institute of Mathematics, supplement 2, pp. S141-S157, 2000.

[17] A. A. Azamov and A. Sh. Kuchkarov, "On controllability and pursuit problems in linear discrete systems," Journal of Computer and Systems Sciences International, vol. 49, no. 3, pp. 360-365, 2010.

[18] G. I. Ibragimov, "Problems of linear discrete games of pursuit," Mathematical Notes, vol. 77, no. 5, pp. 653-662, 2005.

[19] N. Yu. Satimov, B. B. Rikhsiev, and A. A. Khamdamov, "On a pursuit problem for linear differential and discrete n-person games with integral constraints," Matematicheskii Sbornik, vol. 46, no. 4, pp. 459-469, 1982.

[20] N. Yu. Satimov and G. I. Ibragimov, "On a pursuit problem for discrete games with several participants," Izvestiya Vysshikh Uchebnykh Zavedeniŭ. Matematika. Kazanskiı̆ Gosudarstvennyı Universitet, no. 12, pp. 46-57, 2004.

[21] A. A. Azamov, Fundamentals of Theory of Discrete Games, Niso Poligraf, Tashkent, Uzbekistan, 2011, in Russian. 


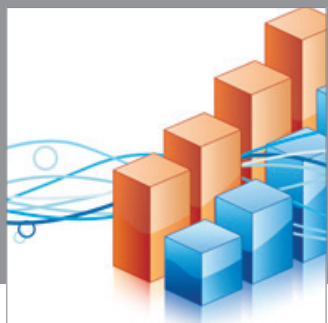

Advances in

Operations Research

mansans

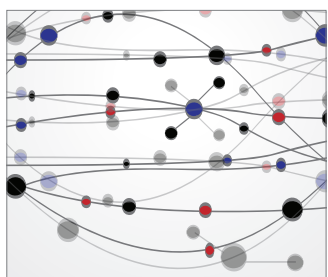

The Scientific World Journal
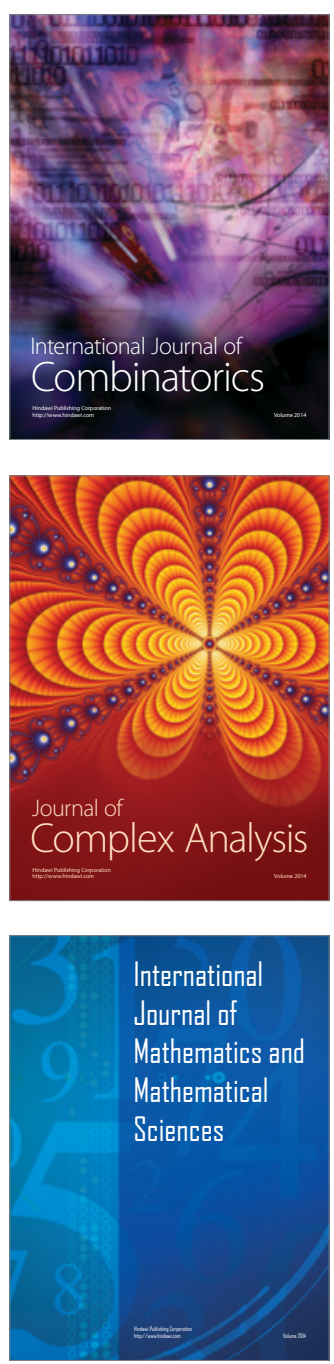
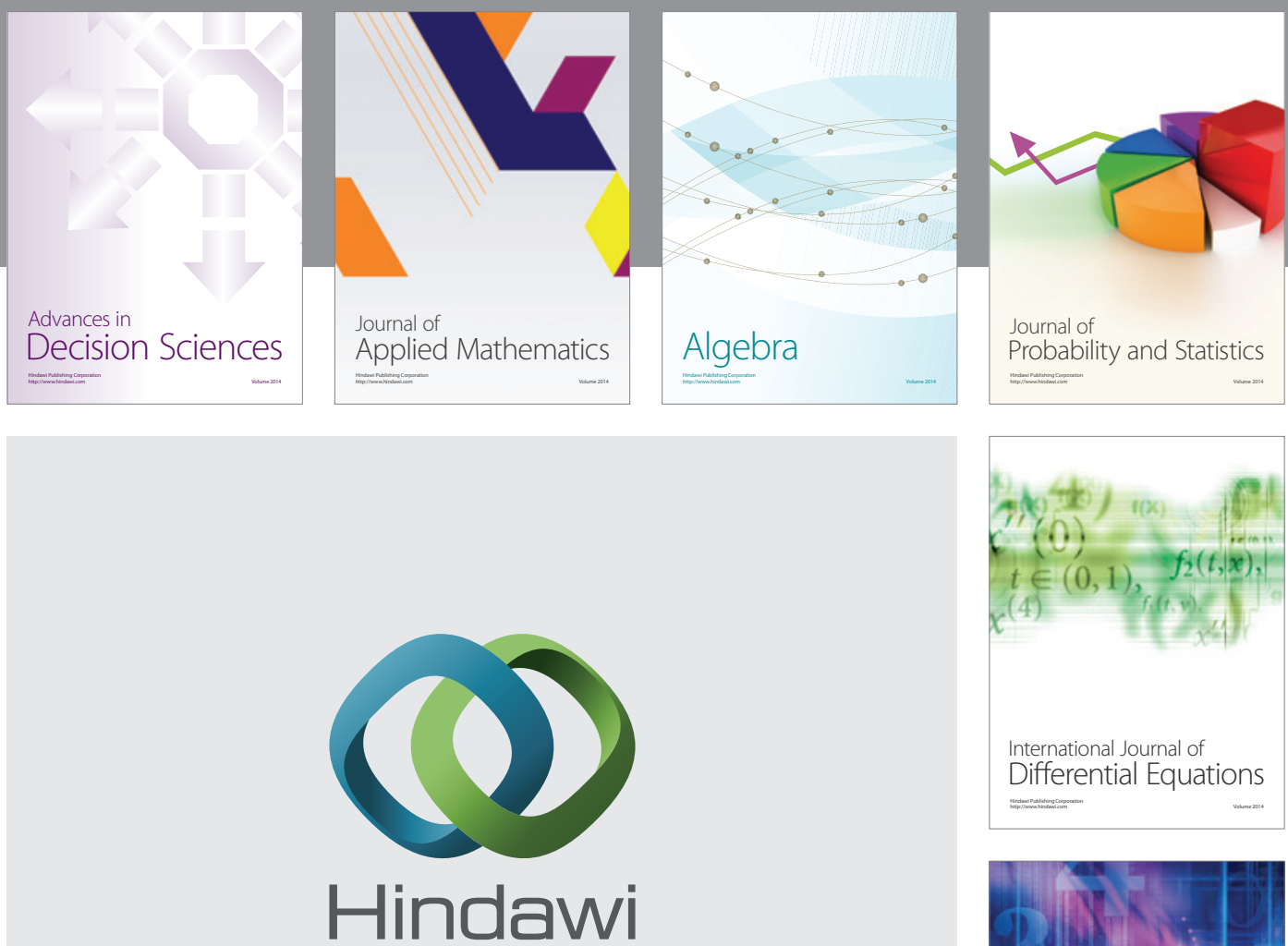

Submit your manuscripts at http://www.hindawi.com
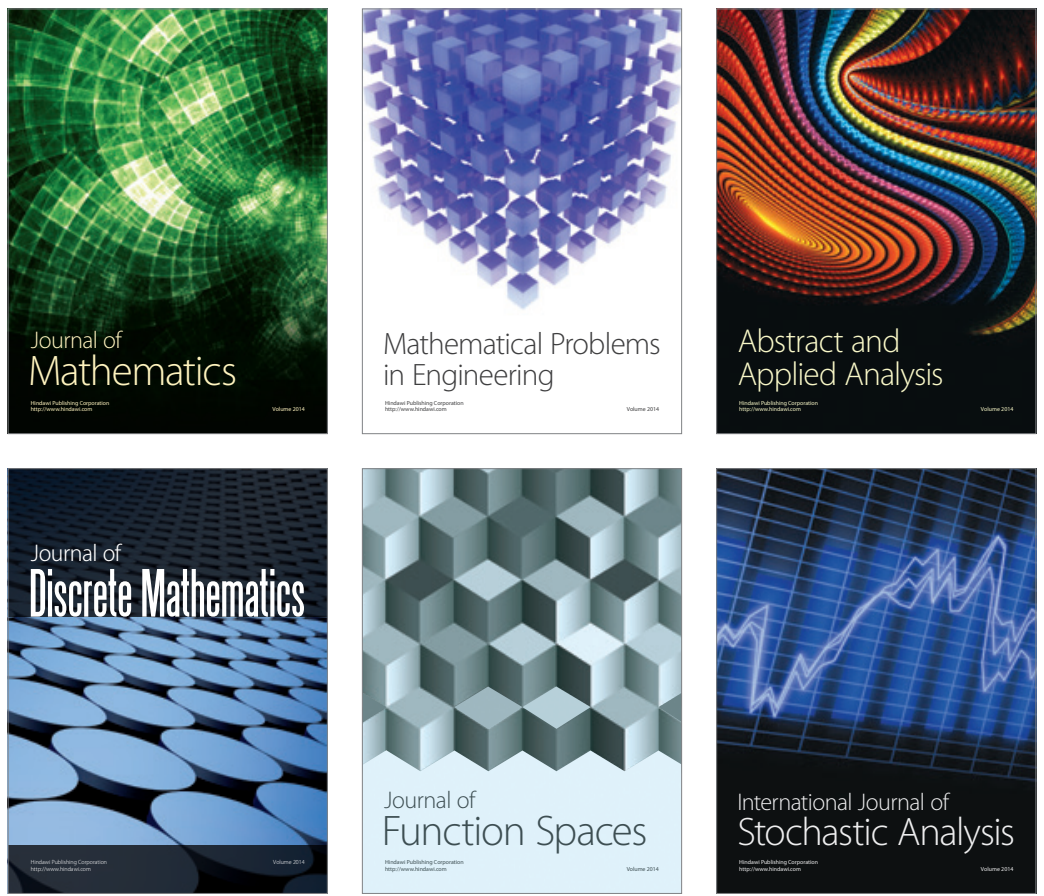

Journal of

Function Spaces

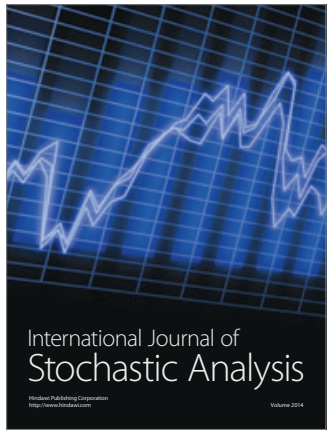

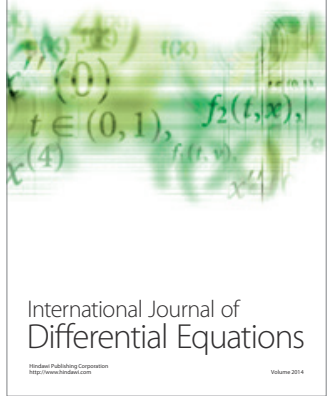
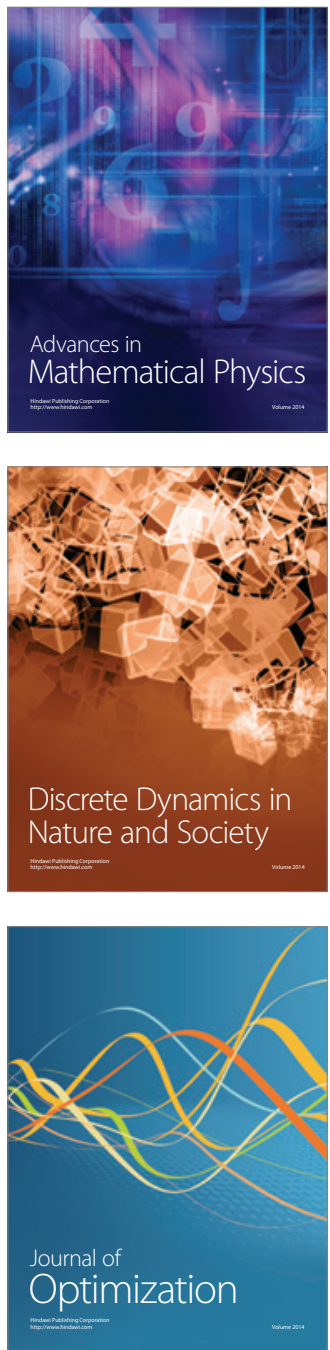